\title{
Joint Design of Spectrum Sensing and Channel Access in Cognitive Radio Networks
}

\author{
Amr A. El-Sherif and K. J. Ray Liu
}

\begin{abstract}
Spectrum sensing is an essential functionality of cognitive radio networks. However, the effect of errors in the spectrum sensing process on the performance of the multiple access layer of both primary and secondary networks has not gained much attention. This paper aims at bridging the gap between the study of spectrum sensing and the multiple access of cognitive radio networks. To achieve this goal we pose and answer the question how the spectrum sensing errors affects the performance of cognitive radio networks from a multiple access protocol design point of view. The negative effects of the spectrum sensing errors on the throughput of both primary and secondary networks are characterized through queuing theory analysis of both networks. To alleviate these negative effects a novel joint design of the spectrum sensing and channel access mechanisms is proposed. This design is based on the observation that, in a binary hypothesis testing problem, the value of the test statistics could be used as a confidence measure for the test outcome. Therefore, this value will be used to define different channel access probabilities for secondary users. Results reveal a significant performance improvement in the maximum stable throughput of both primary and secondary networks by virtue of the proposed technique.
\end{abstract}

Index Terms-Cognitive radio, soft sensing, multiple access.

\section{INTRODUCTION}

C OGNITIVE radio is a promising technology aiming at better utilization of available channel resources[1]. Cognitive radio prescribes the coexistence of licensed (or primary) and unlicensed (secondary or cognitive) radio nodes on the same bandwidth. While the first group is allowed to access the spectrum any time, the second seeks opportunities for transmission by exploiting the idle periods of primary nodes. In [2] and [3] the cognitive radio problem was investigated from an information theoretic standpoint, where the cognitive transmitter is assumed to transmit at the same time and on the same bandwidth of the primary link, being able to mitigate its interference toward the primary through complex precoding techniques that are based on the perfect prior information about the signal transmitted by the primary. Centralized and decentralized protocols at the media access control (MAC) layer aiming at minimizing secondary nodes interference with primary transmissions have been studied in [4] and [5] by modeling the radio channel as either busy (i.e., the primary user is active) or available (i.e., the primary user

Manuscript received January 30, 2010; revised September 7, 2010 and February 5, 2011; accepted February 8, 2011. The associate editor coordinating the review of this paper and approving it for publication was E. Hossain.

A. A. El-Sherif is with the Department of Electrical Engineering, Alexandria University, Alexandria 21544, Egypt (e-mail: amr.elsherif@ieee.org).

K. J. R. Liu is with the Department of Electrical and Computer Engineering, University of Maryland, College Park, MD 20742, USA (e-mail: kjrliu@umd.edu).

Digital Object Identifier 10.1109/TWC.2011.032411.100131 is idle) according to a Markov chain. The question of how to efficiently and fairly share the spectrum among multiple dissimilar users has been addressed from a game theoretic viewpoint in [6], [7].

Spectrum sensing is an essential functionality of cognitive radios, since the devices need to reliably detect weak primary signals of possibly unknown types [8]. Various spectrum sensing techniques exist, such as energy detection [9], matched filter coherent detection [10], and cyclostationary feature detection [11]. While these classic signal detection techniques are well known, detecting primary transmitters in a dynamic wireless environment with noise uncertainty, shadowing, and fading is a challenging problem as articulated in [12]. To improve detection accuracy, cooperative spectrum sensing has been proposed [12], [13], [14]. The basic idea is to overcome shadowing and multipath fading by allowing neighboring secondary users to exchange sensing information through a dedicated control channel. In [15], two decision-combining approaches were studied: hard decision with the AND logic operation and soft decision using the likelihood ratio test [16]. It was shown that the soft decision combination of spectrum sensing results yields gains over hard decision combining. In [17], the authors exploited the fact that summing signals from two secondary users can increase the signal-to-noise ratio (SNR) and detection reliability if the signals are correlated.

One of the assumptions in many of the works on cognitive radios and dynamic spectrum sharing [4], [18]-[20], is that the effects of the sensing mechanism on the performance of the channel access mechanism is negligible. In other words, the assumption is made that secondary nodes have perfect knowledge of whether primary nodes are active or not. This assumption allows researchers to deal with the two main problems of dynamic spectrum sharing and cognitive radios separately. The first problem is the design of highly sensitive detectors to accurately detect the presence or absence of transmissions from primary users. And the second problem is spectrum access coordination between different secondary users while limiting the level of interference to the primary system. In the literature, this problem has been addressed on a negotiating/pricing basis [6], [21]-[25] or an opportunistic basis [26], [27].

In this paper we consider the effects of spectrum sensing errors on the performance of cognitive radio networks. While the issue of spectrum sensing errors has been investigated at the physical layer [8], [12], [13], [28], [29], cognitive multiple access design in the presence of sensing errors has received little attention. We specifically try to answer the questions: How does the spectrum sensing errors affect the 


\begin{tabular}{|l|l|}
\hline Sensing & Data \\
\hline
\end{tabular}

Fig. 1. Time slot structure, showing the sensing period used by secondary users.

performance of the cognitive radio network from a multiple access protocol design point of view? And, how can the joint design of spectrum sensing and access mechanisms mitigate the negative effects of sensing errors? To answer the questions posed above, this paper starts by studying the effects of channel sensing errors on the performance from the point of view of multiple access layer measures. This is achieved through a queueing theoretical analysis of the stability regions of both primary and secondary networks. The stability region is characterized for different operating points on the receiver operating characteristic (ROC) of the energy detector based spectrum sensor. The results reveal a significant reduction in the stability region of both networks due to sensing errors.

To mitigate the negative effects of sensing errors, we propose a novel joint design of the spectrum sensing and access mechanisms. The design is based on the observation that, in a binary hypothesis testing problem, the value of the test statistics could be used as a measure of how confident we are in the test outcome. The further the value of the test statistics is from the decision threshold, the more confident we are that the decision is correct. Therefore, instead of using the hard decisions of the spectrum sensor to decide whether to access the channel or not, a secondary user can have different access probabilities for different values of the test statistics. For instance, the access probability could be higher for the values of the test statistics further away from the decision threshold, and vice versa. Using this technique, one can set the target false alarm probability as low as possible for the secondary nodes not to overlook spectrum opportunities. At the same time a low probability of collision with primary users could be maintained since the access probability can be set to an arbitrarily low value near the decision threshold, which is not the case with conventional designs, since lowering the false alarm probability results in an increased probability of missed detections, hence increased probability of collision.

The rest of the paper is organized as follows, the network and channel models used are described in section II. The effects of sensing errors on the performance of both primary and secondary networks are characterized in section III. In section IV we propose our novel joint design scheme for the channel sensing and channel access mechanisms, and its performance is thoroughly studied and compared to other schemes. Finally, this work is concluded in section V.

\section{Channel And Queuing Models}

We consider the uplink of a TDMA cellular network as the primary network. The primary network consists of $M_{p}$ source nodes numbered $1,2, \ldots, M_{p}$ communicating with a base station (BS) $d_{p}$. A secondary network, consisting of $M_{s}$ nodes numbered $1,2, \ldots, M_{s}$, tries to exploit the unutilized channel resources to communicate their own data packets using slotted ALOHA as their multiple access protocol. Let
$\mathcal{M}_{p}=1,2, \ldots, M_{p}$ denote the set of primary nodes, and $\mathcal{M}_{s}=1,2, \ldots, M_{s}$ denote the set of secondary nodes.

Secondary nodes independently exploit instantaneous spectrum opportunities in the channel (in the form of idle time slots in our case). At the beginning of each slot, a secondary node with data to transmit resorts to a spectrum sensor is used to detect the state of the medium (idle or busy). Based on the sensing outcomes, the secondary node decides whether to access the channel or not. At the end of the slot, the receiver acknowledges each successful transmission. The basic slot structure is illustrated in Fig. 1, in which a sensing period followed by the data transmission period are shown.

\section{A. Channel Model}

The wireless channel between a node and its destination is modeled as a Rayleigh flat fading channel with additive white Gaussian noise. The signal received at a receiving node $j$ from a transmitting node $i$ at time $t$ is then modeled as

$$
y_{i j}^{t}=\sqrt{G_{i} \rho_{i j}^{-\gamma}} h_{i j}^{t} x_{i}^{t}+n_{j}^{t},
$$

where $G_{i}$ is the transmitting power, assumed to be the same for all nodes, $\rho_{i j}$ denotes the distance between the two nodes, $\gamma$ the path loss exponent, $h_{i j}^{t}$ is the channel fading coefficient between nodes $i$ and $j$ at time $t$ and is modeled as an i.i.d zero mean, circularly symmetric complex gaussian random process with unit variance. The term $x_{i}^{t}$ denotes the transmitted signal which has an average unit power and is assumed to be drawn from a constant modulus constellation with zero mean (M-ary PSK for instance). The i.i.d additive white Gaussian noise processes $n_{j}^{t}$ have zero mean and variance $N_{0}$. Since the arrivals, the channel gains, and the additive noise processes are all assumed stationary, we can drop the index $t$ without loss of generality.

Success and failure of packet reception is characterized by outage events and outage probabilities, which is defined as follows. For a targeted signal-to-noise (SNR) ratio $\delta$ (called outage $S N R$ [30]), if the received SNR as a function of the fading realization $h$ is given by $\operatorname{SNR}(h)$, then the outage event $O$ is the event that $\operatorname{SNR}(h)<\delta$, and $\operatorname{Pr}\{\operatorname{SNR}(h)<\delta\}$ denotes the outage probability. This definition is equivalent to the capture model in [31], [32]. The SNR threshold $\delta$ is a function of different parameters in the communication system; it is a function of the application, the data rate, the signalprocessing applied at encoder/decoder sides, error-correction codes, and other factors. For example, varying the data rate and fixing all other parameters, the required SNR threshold $\delta$ to achieve certain system performance is a monotonically increasing function of the data rate. Also, increasing the signal processing and encoder/decoder complexity in the physical layer reduces the required SNR threshold $\delta$ for a required system performance.

For the channel model in (1) the received SNR of a signal transmitted between any two nodes $i$ and $j$ can be specified as follows

$$
\mathrm{SNR}_{i j}=\frac{\left|h_{i j}\right|^{2} \rho_{i j}^{-\gamma} G_{i}}{N_{0}}
$$




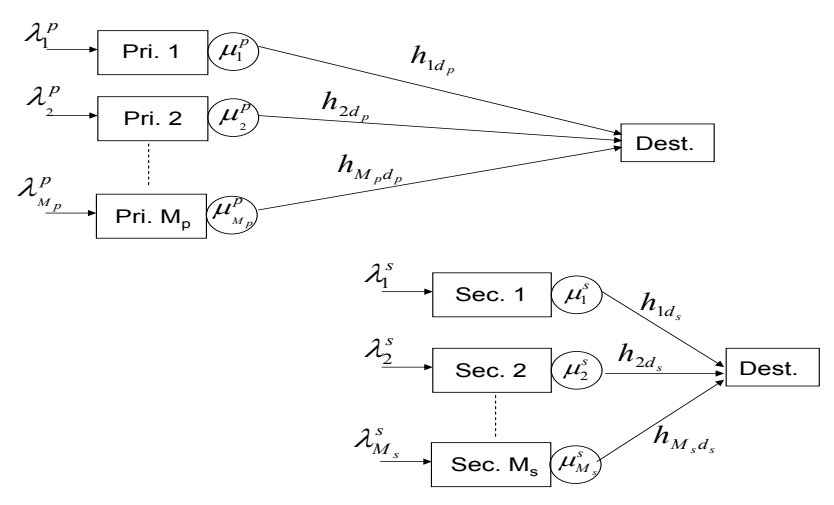

Fig. 2. Network queuing and channel model.

Since the SNR in (2) is a monotone function of $\left|h_{i j}\right|^{2}$, the outage event for an outage SNR $\delta$ is equivalent to

$$
\left\{h_{i j}: \operatorname{SNR}_{i j}<\delta\right\}=\left\{h_{i j}:\left|h_{i j}\right|^{2}<\frac{\delta N_{0} \rho_{i j}^{\gamma}}{G_{i}}\right\} .
$$

Accordingly, and knowing that $\left|h_{i j}\right|^{2}$ has an exponential distribution, the outage probability is

$$
P_{i j}^{o}=\operatorname{Pr}\left\{\left|h_{i j}\right|^{2}<\frac{\delta N_{0} \rho_{i j}^{\gamma}}{G_{i}}\right\}=1-\exp \left(-\frac{\delta N_{0} \rho_{i j}^{\gamma}}{G_{i}}\right) .
$$

In the case of simultaneous transmissions from two or more nodes, two models could be used to deal with this scenario. The first is the interference channel model, in which one node's signal is considered as interference to the other node's signal. Under this model, one of the transmitted signals could be decoded correctly at the BS given high enough SNR. The second model is the collision channel model, in which all simultaneous transmissions are assumed to be lost with no regard to the received SNR level of individual signals. In this paper we adopt the more restrictive collision channel model, and assume that all packets are lost in the case of a collision between a primary transmission and a secondary transmission, or between two or more secondary transmissions.

Furthermore, we assume that whenever there is a collision between a primary transmission and a secondary transmission, or between two or more secondary transmissions, all the packets involved are lost.

\section{B. Queuing Model}

Each primary or secondary node has an infinite buffer for storing fixed length packets (see Fig. 2). The channel is slotted in time and a slot duration equals the packet transmission time. The arrivals at the $i^{\text {th }}$ primary node's queue $\left(i \in \mathcal{M}_{p}\right)$, and the $j^{\text {th }}$ secondary node's queue $\left(i \in \mathcal{M}_{s}\right)$ are Bernoulli random variables, i.i.d from slot to slot with mean $\lambda_{i}^{p}$ and $\lambda_{j}^{s}$, respectively. Hence, the vector $\Lambda=\left[\lambda_{1}^{p}, \ldots, \lambda_{M_{p}}^{p}, \lambda_{1}^{s}, \ldots, \lambda_{M_{s}}^{s}\right]$ denotes the average arrival rates. Arrival processes are assumed to be independent from one node to another.

Primary nodes access the channel by dividing the channel resources, time in this case, among them, hence, each node is allocated a fraction of the time. Let $\boldsymbol{\Omega}_{\mathbf{p}}=\left[\omega_{1}^{p}, \omega_{2}^{p}, \ldots, \omega_{M_{p}}^{p}\right]$ denote a resource-sharing vector, where $\omega_{i}^{p} \geq 0$ is the fraction of time allocated to node $i \in \mathcal{M}_{p}$, or it can represent the probability that node $i$ is allocated the whole time slot [33]. The set of all feasible resource-sharing vectors is specified as follows

$$
\digamma_{p}=\left\{\boldsymbol{\Omega}_{\mathbf{p}}=\left(\omega_{1}^{p}, \omega_{2}^{p}, \ldots, \omega_{M_{p}}^{p}\right) \in \Re^{+M_{p}}: \sum_{i \in \mathcal{M}_{p}} \omega_{i}^{p}<=1\right\} .
$$

In this work we are considering the case where no time slot is left unassigned to a primary node, in other words $\mathcal{M}_{p} \omega_{i}^{p}=1$. In a communication network, the stability of the network's queues is a fundamental performance measure. Stability can be loosely defined as having a certain quantity of interest kept bounded. In our case, we are interested in the queue size being bounded. More rigourously, stability can be defined as follows (for the primary network alone). Denote the queue sizes of the transmitting nodes at any time $t$ by the vector $\mathbf{Q}^{t}=\left[Q_{i}^{t}, i \in\right.$ $\left.\mathcal{M}_{p}\right]$. We adopt the following definition of stability used in [34]. Queue $i \in \mathcal{M}_{p}$ is stable if,

$$
\lim _{t \rightarrow \infty} \operatorname{Pr}\left\{Q_{i}^{t}<x\right\}=F(x) \text { and } \lim _{x \rightarrow \infty} F(x)=1 .
$$

If $\lim _{x \rightarrow \infty} \lim _{t \rightarrow \infty} \inf \operatorname{Pr}\left\{Q_{i}^{t}<x\right\}=1$, the queue is called substable. From the definition, if a queue is stable then it is also substable. If a queue is not substable, then it is unstable. An arrival rate vector $\Lambda=\left[\lambda_{1}^{p}, \ldots, \lambda_{M_{p}}^{p}\right]$ is said to be stable if there exists a resource sharing vector $\Omega_{p} \in \digamma_{p}$ such that all the queues are stable. The multidimensional stochastic process $\mathbf{Q}^{t}$ can be easily shown to be an irreducible and aperiodic discrete-time Markov chain process with a countable number of states and state space $\in \mathrm{Z}_{+}^{M_{p}}$ [35]. For such a Markov chain, the process is stable if and only if there exists a positive probability for every queue being empty [36], i.e.,

$$
\lim _{t \rightarrow \infty} \operatorname{Pr}\left\{Q_{i}^{t}=0\right\}>0, \quad i \in \mathcal{M}_{p} .
$$

If the arrival and service processes of a queueing system are strictly stationary, then one can apply Loynes's theorem to check for stability conditions [37]. This theorem states that if the arrival process and the service process of a queueing system are strictly stationary, and the average arrival rate is less than the average service rate, then the queue is stable; if the average arrival rate is greater than the average service rate then the queue is unstable.

\section{Effect of Sensing Errors on Performance}

\section{A. Spectrum Sensing}

In this section we will study the effects of sensing errors on the performance of both primary and secondary networks. because of its simplicity and ability to locate spectrum occupancy information quickly, non-coherent energy detected will be adopted in our study of the effect of sensing errors on cognitive radio's performance. Detection of the presence of the $i^{t h}$ primary node by the $j^{t h}$ secondary node can be formulated as a binary hypothesis test as follows,

$$
\begin{aligned}
& \mathcal{H}_{0}: y_{i j}^{t}=n_{j} \\
& \mathcal{H}_{1}: y_{i j}^{t}=\sqrt{G_{i} \rho_{i j}^{-\gamma}} h_{i j}^{t} x_{i}+n_{j} .
\end{aligned}
$$


The null hypothesis $\mathcal{H}_{0}$ represents the absence of the primary node, hence a transmission opportunity to the secondary node. And the alternative hypothesis $\mathcal{H}_{1}$ represents a transmitting primary node.

The performance of the spectrum sensor is characterized by the two types of errors and their probabilities, (i) false alarms having probability $\alpha$, (ii) and missed detections having probability $\beta$, with

$$
\begin{gathered}
\alpha \triangleq \operatorname{Pr}\left\{\text { decide } \mathcal{H}_{1} \mid \mathcal{H}_{0} \text { is true }\right\} \\
\beta \triangleq \operatorname{Pr}\left\{\text { decide } \mathcal{H}_{0} \mid \mathcal{H}_{1} \text { is true }\right\}
\end{gathered}
$$

The false alarm type of errors where an idle channel is erroneously detected as busy does not incur performance degradation on the primary system, but lowers the potential channel utilization of secondary nodes. On the other hand, the missed detection events, where a secondary node fails to detect a primary transmission, will result in a collision between primary and secondary transmissions. Therefore, miss detection events will negatively impact the performance of the primary system.

With the assumption that secondary nodes do not have prior knowledge of primary activity patterns, the probability of miss detection $\beta$ could be minimized subject to the constraint that the probability of false alarm is no larger than a given value $\alpha$ using the optimal Neyman-Pearson (NP) detector [10].

From the received signal model of (1), it follows that under hypothesis $\mathcal{H}_{0}$ the received signal $y_{i j}$ is a complex Gaussian random variable with zero mean and variance $\sigma_{0}^{2}=N_{0}$, and under hypothesis $\mathcal{H}_{1}, y_{i j}$ is a complex Gaussian random variable with zero mean and variance

$$
\sigma_{i j}^{2}=G_{i} \rho_{i j}^{-\gamma}+N_{0} .
$$

Therefore, the likelihood ratio test for the optimal NP detector can be written as follows,

$$
\begin{aligned}
\Lambda\left(y_{i j}\right) & =\frac{\operatorname{Pr}\left\{y_{i j} \mid \mathcal{H}_{1}\right\}}{\operatorname{Pr}\left\{y_{i j} \mid \mathcal{H}_{0}\right\}}=\frac{\frac{1}{\pi \sigma_{i j}^{2}} e^{-\frac{\left\|y_{i j}\right\|^{2}}{\sigma_{i j}^{2}}}}{\frac{1}{\pi \sigma_{0}^{2}} e^{-\frac{\left\|y_{i j}\right\|^{2}}{\sigma_{0}^{2}}}} \\
= & \frac{\sigma_{0}^{2}}{\sigma_{i j}^{2}} e^{-\left\|y_{i j}\right\|^{2}\left[\frac{1}{\sigma_{i j}^{2}}-\frac{1}{\sigma_{0}^{2}}\right]} \gtrless \mathcal{H}_{0} \eta^{\prime},
\end{aligned}
$$

which can be simplified to

$$
\left\|y_{i j}\right\|^{2} \gtrless \mathcal{H}_{\mathcal{H}_{0}} \frac{\eta^{\prime}-\log \frac{\sigma_{0}^{2}}{\sigma_{i j}^{2}}}{\frac{1}{\sigma_{0}^{2}}-\frac{1}{\sigma_{i j}^{2}}}=\eta .
$$

From (12), the spectrum sensing problem has been reduced to a simple comparison of the received signal energy $\left\|y_{i j}\right\|^{2}$ to a threshold $\eta$. The optimum threshold could then be calculated through the constraint on the false alarm probability. We first note that, from the received signal model of (1), $\left\|y_{i j}\right\|^{2}$ is exponentially distributed with parameter $1 / 2 \sigma_{i j}^{2}$ and $1 / 2 \sigma_{0}^{2}$, under $\mathcal{H}_{1}$ and $\mathcal{H}_{0}$, respectively. Therefore, the false alarm probability is

$$
\alpha=\operatorname{Pr}\left\{|| y_{i j} \|^{2}>\eta \mid \mathcal{H}_{0}\right\}=e^{-\frac{\eta}{2 \sigma_{0}^{2}}} .
$$

From which

$$
\eta=-2 \sigma_{0}^{2} \log (\alpha)
$$

Finally, the probability of misdetection is

$$
\beta=\operatorname{Pr}\left\{|| y_{i j} \|^{2}<\eta \mid \mathcal{H}_{1}\right\}=1-e^{-\frac{\eta}{2 \sigma_{1}^{2}}}=1-e^{-\frac{\sigma_{0}^{2} \log (\alpha)}{\sigma_{i j}^{2}}} .
$$

It is noted that in the design above, the spectrum sensor has based its detection on a single sample of the received signal. Increasing the number of samples will of course increase the reliability of the sensing process. However, we limited ourselves to this design for the purpose of mathematical tractability as it will be clear later.

\section{B. Performance Analysis}

To analyze the effect of sensing errors on the cognitive radio system, we adopt the stability regions of the primary and secondary networks as the performance measure. We will begin by characterizing the stability region for the ideal system with no sensing errors to form a base for comparison.

1) System with Perfect Sensing: We start by characterizing the stability region for the primary system of queues. Since the primary network employs TDMA as a multiple access protocol, it follows directly from Loynes's theorem [37], that the primary nodes' stability region $\mathcal{R}_{p}$ is defined as

$$
\begin{array}{r}
\mathcal{R}_{p}=\left\{\left(\lambda_{1}^{p}, \ldots, \lambda_{M_{p}}^{p}\right) \in R^{+M_{p}}: \lambda_{i}^{p}<\omega_{i}^{p}\left(1-P_{i}^{o p}\right),\right. \\
\left.\forall i \in \mathcal{M}_{p},\left(\omega_{1}^{p}, \ldots, \omega_{M_{p}}^{p}\right) \in \digamma_{p}\right\},
\end{array}
$$

which can be easily shown to be equivalent to

$$
\mathcal{R}_{p}=\left\{\left(\lambda_{1}^{p}, \ldots, \lambda_{M_{p}}^{p}\right) \in R^{+M_{p}}: \sum_{i \in \mathcal{M}_{p}} \frac{\lambda_{i}^{p}}{1-P_{i}^{o p}} \leq 1\right\},
$$

where $P_{i}^{o p}$ is the outage probability of the link between the $i^{t h}$ primary node and the primary destination.

For the secondary network, we recall that secondary nodes employ slotted ALOHA to share idle time slots among themselves. Therefore, when an idle time slot is detected, the $i^{t h}$ secondary node will try to transmit the packet at the head of its queue (if any) with access probability $p_{i}^{a}$. We note that, because of the possible collisions between secondary transmissions, secondary nodes' queues are interacting. In other words, the service rate of a given queue is dependent on the state of all other queues, i.e., whether they are empty or not. Studying the stability conditions for interacting queues is a difficult problem that has been addressed for ALOHA systems [38], [36] [39]. The concept of dominant systems was introduced and employed in [38] to help find bounds on the stability region of ALOHA with collision channel. The dominant system in [38] was defined by allowing a set of terminals with no packets to transmit to continue transmitting dummy packets. In this manner, the queues in the dominant system stochastically dominate the queues in the original system. Or in other words, with the same initial conditions for queue sizes in both the original and dominant systems, the queue sizes in the dominant system are not smaller than those in the original system. Therefore, stability of the dominant 
system is a sufficient but not necessary condition for the stability of the original system of queues. Thus, the stability region obtained through the dominant system analysis is an inner bound to the actual system's stability region. In the following discussions any mention to the stability region refers to this inner bound obtained through the dominant system analysis.

To study the stability of the interacting system of queues consisting of secondary nodes' queues, we make use of the dominant system approach to decouple the interaction between queues. We define the dominant system as follows:

- Arrivals at each queue in the dominant system are the same as in the original system.

- Time slots assigned to primary node $i \in \mathcal{M}_{p}$ are identical in both systems.

- The outcomes of the "coin tossing" (that determines transmission attempts of secondary nodes) in every slot are the same.

- Channel realizations for both systems are identical, i.e., if a given channel is in outage in the original system then it is also in outage in the dominant system.

- The noise generated at the receiving ends of both systems is identical.

- In the dominant system, secondary nodes attempt to transmit dummy packets when their queues are empty.

The service process of a secondary node depends on the idle time slots unused by the primary nodes. Therefore, the service process of the $j^{\text {th }}$ secondary node can be modeled as

$Y_{s j}^{t}=\sum_{i \in \mathcal{M}_{p}} \mathbf{1}\left[A_{i}^{t} \bigcap\left\{Q_{i}^{t}=0\right\} \bigcap \overline{O_{j}^{s t}} \bigcap P_{j}^{a} \bigcap_{k \in \mathcal{M}_{s} \backslash j}\left\{\overline{P_{k}^{a}}\right\}\right]$

where $A_{i}^{t}$ denotes the event that slot $t$ is assigned to primary node $i,\left\{Q_{i}^{t}=0\right\}$ denotes the event that this node's queue is empty, i.e., the node has no packets to transmit, and according to Little's theorem [40] it has probability $\left(1-\lambda_{i}^{p} / \mu_{i}^{p}\right)$. From the definition in (16),

$$
\mu_{i}^{p}=\omega_{i}^{p}\left(1-P_{i}^{o p}\right) .
$$

Event $P_{j}^{a}$ is the event that the $j^{t h}$ secondary node has permission to transmit, which has probability $p_{j}^{a}$. Therefore, the event $P_{j}^{a} \bigcap_{k \in \mathcal{M}_{s} \backslash j}\left\{\overline{P_{k}^{a}}\right\}$ is that only the $j^{t h}$ secondary node is transmitting in the current time slot; otherwise a collision will occur and all packets involved will be lost. Finally, $\overline{O_{j}^{s t}}$ denotes the event that link between the $j^{\text {th }}$ secondary node and its destination is not in outage.

Assuming that primary queues are stable, then they offer stationary empty slots. Also the channel statistics are stationary; hence, the secondary service process is stationary. The average secondary service rate is then given by

$$
\begin{array}{r}
\mu_{j}^{s}=E\left[Y_{s j}^{t}\right]=\left(1-\sum_{i \in \mathcal{M}_{p}} \frac{\lambda_{i}^{p}}{1-P_{i}^{o p}}\right)\left(1-P_{j}^{o s}\right) p_{i}^{a} \\
\times \prod_{k \in \mathcal{M}_{s} \backslash j}\left(1-p_{k}^{a}\right),
\end{array}
$$

where $P_{j}^{o s}$ is the outage probability of the link between any primary node and its destination.
Using Loyne's theorem along with (20), and from (16), the stability region of the system defined by the primary and secondary nodes can be written as

$$
\begin{aligned}
\mathcal{R}= & \mathcal{R}_{p} \bigcap \mathcal{R}_{s}=\left\{\left(\lambda_{1}^{p}, \ldots, \lambda_{M_{p}}^{p}, \lambda_{1}^{s}, \ldots, \lambda_{M_{s}}^{s}\right) \in R^{+\left(M_{p}+M_{s}\right)}:\right. \\
& \sum_{i \in \mathcal{M}_{p}} \frac{\lambda_{i}^{p}}{1-P_{i}^{o p}} \leq 1, \lambda_{j}^{s}<\left(1-\sum_{i \in \mathcal{M}_{p}} \frac{\lambda_{i}^{p}}{1-P_{i}^{o p}}\right) \\
& \left.\left(1-P_{j}^{o s}\right) p_{i}^{a} \prod_{k \in \mathcal{M}_{s} \backslash j}\left(1-p_{k}^{a}\right), \forall j \in \mathcal{M}_{s}\right\} .
\end{aligned}
$$

It is clear from (16) and (20) that the primary network stability is completely independent from the secondary network operation. The secondary network stability is dependent on the primary network through the condition of empty primary queues for secondary queues service to take place.

2) System with Non-Perfect Sensing: In the case of nonperfect sensing, the events of misdetection will result in simultaneous primary and secondary transmissions leading to collisions and data loss. Because of these collision events, primary and secondary queues are now interacting. To analyze this interacting system of queues, we will resort to the dominant system discussed above.

Under the dominant system in which secondary nodes attempt to transmit dummy packets if their queues are empty, the service process of the $i^{\text {th }}$ primary node is defined as follows,

$$
Y_{p i}^{t}=\mathbf{1}\left[A_{i}^{t} \bigcap \overline{O_{i}^{p t}} \bigcap_{j \in \mathcal{M}_{s}}\left\{\overline{\mathcal{B}_{j}^{i} \bigcap P_{j}^{a}}\right\}\right],
$$

where $\mathcal{B}_{j}^{i}$ is the event of a misdetection of primary node $i$ by secondary node $j$, and has a probability $\beta_{j}^{i}$. Therefore, $\overline{\mathcal{B}_{j}^{i} \cap P_{j}^{a}}$ is the complement of the event that a secondary node misdetects the primary activity and has access permission, hence causing a collision with the primary node. From the definition of the service process, it follows that the average primary service rate is defined as

$$
\mu_{i}^{p}=E\left[Y_{p i}^{t}\right]=\omega_{i}^{p}\left(1-P_{i}^{o p}\right) \prod_{j \in \mathcal{M}_{s}}\left(1-\beta_{j}^{i} p_{j}^{a}\right) .
$$

Similarly, the service process of the $j^{\text {th }}$ secondary node can be written as

$$
\begin{array}{r}
Y_{s j}^{t}=\sum_{i \in \mathcal{M}_{p}} \mathbf{1}\left[A_{i}^{t} \bigcap\left\{Q_{i}^{t}=0\right\} \bigcap \overline{O_{j}^{s t}} \bigcap \overline{\mathcal{A}_{j}} \bigcap P_{j}^{a}\right. \\
\left.\bigcap_{k \in \mathcal{M}_{s} \backslash j}\left\{\overline{\overline{\mathcal{A}_{k}} \bigcap P_{k}^{a}}\right\}\right],
\end{array}
$$

where $\mathcal{A}_{j}$ is the event of false alarm at the $j^{\text {th }}$ secondary node and has a probability $\alpha_{j}$. The above definition of the secondary service process accounts for the fact that, for a secondary node to gain uncontested access to an idle time slot, it should correctly identify the slot as idle and have access permission. At the same time all other secondary nodes do not access the channel during that time slot, which is represented as $\left\{\overline{\overline{\mathcal{A}_{k}} \cap P_{k}^{a}}\right\}$, which is the complement of the aforementioned 
event. From this definition, the average secondary service rate is then

$$
\begin{array}{r}
\mu_{j}^{s}=E\left[Y_{s j}^{t}\right]=\left(1-\sum_{i \in \mathcal{M}_{p}} \frac{\lambda_{i}^{p}}{\left(1-P_{i}^{o p}\right) \prod_{j \in \mathcal{M}_{s}}\left(1-\beta_{j}^{i} p_{j}^{a}\right)}\right) \\
\times\left(1-\alpha_{j}\right) p_{j}^{a}\left(1-P_{j}^{o s}\right) \prod_{k \in \mathcal{M}_{s} \backslash j}\left(1-\left(1-\alpha_{k}\right) p_{k}^{a}\right) .
\end{array}
$$

Using Loyne's theorem along with (23), and (25), the stability region for a given secondary access probability vector $\mathbf{p}^{a}=$ $\left(p_{1}^{a}, p_{2}^{a}, \ldots, p_{M_{s}}^{a}\right)$ of the system defined by the primary and secondary nodes can be written as

$$
\begin{aligned}
& \mathcal{R}\left(\mathbf{p}^{a}\right)=\left\{\left(\lambda_{1}^{p}, \ldots, \lambda_{M_{p}}^{p}, \lambda_{1}^{s}, \ldots, \lambda_{M_{s}}^{s}\right) \in R^{+\left(M_{p}+M_{s}\right)}:\right. \\
& \sum_{i \in \mathcal{M}_{p}} \frac{\lambda_{i}^{p}}{\left(1-P_{i}^{o p}\right) \prod_{j \in \mathcal{M}_{s}}\left(1-\beta_{j}^{i} p_{j}^{a}\right)}<1, \\
& \lambda_{j}^{s}<\left(1-\sum_{i \in \mathcal{M}_{p}} \frac{\lambda_{i}^{p}}{\left(1-P_{i}^{o p}\right) \prod_{j \in \mathcal{M}_{s}}\left(1-\beta_{j}^{i} p_{j}^{a}\right)}\right) \\
& \times\left(1-\alpha_{j}\right) p_{j}^{a}\left(1-P_{j}^{o s}\right) \\
&\left.\times \prod_{k \in \mathcal{M}_{s} \backslash j}\left(1-\left(1-\alpha_{k}\right) p_{k}^{a}\right), \forall j \in \mathcal{M}_{s}\right\},
\end{aligned}
$$

and the maximum stability region can be determined by taking the union over all possible values of $\mathbf{p}^{a}$ as follows,

$$
\mathcal{R}=\bigcup_{\mathbf{p}^{a} \in[0,1]^{M_{s}}}\left\{\mathcal{R}\left(\mathbf{p}^{a}\right)\right\}
$$

Degradation in performance due to sensing errors is clear from (23) and (25). It is seen that the average primary service rate is a monotonically decreasing function of the misdetection probability $\beta$. Therefore, in order not to severely degrade primary performance, where such a degradation contradicts the principle that presence of the secondary system should be transparent to the primary system, spectrum sensors should be designed with the lowest possible $\beta$. Moreover, with lower primary service rate, the channel will busy with higher probability, which negatively affects secondary nodes, since there will be no enough idle time slots for them to use. But, decreasing $\beta$ comes at the expense of a higher false alarm rate $\alpha$, which from (25) will degrade the performance of secondary nodes.

\section{Numerical Results}

To see how non-perfect spectrum sensing affects the stability region of the system of primary and secondary nodes, we consider a network with $M_{p}=2$ primary nodes and $M_{s}=2$ secondary nodes. Nodes are uniformly distributed over a square region with $250 \mathrm{~m}$ edges. Primary and secondary destination are located at the center of the square region. SNR threshold is $15 \mathrm{~dB}$, transmit power is $100 \mathrm{~mW}$, path loss exponent $\gamma=3.7$, and noise power $N_{0}=10^{-11} \mathrm{~W}$. Results are obtained by averaging over 20 independent realizations. For ease of illustration we plot the aggregate primary arrival rate $\lambda^{p}=\sum_{i} \lambda_{i}^{p}$ and aggregate secondary arrival rate $\lambda^{s}=\sum_{j} \lambda_{j}^{s}$.

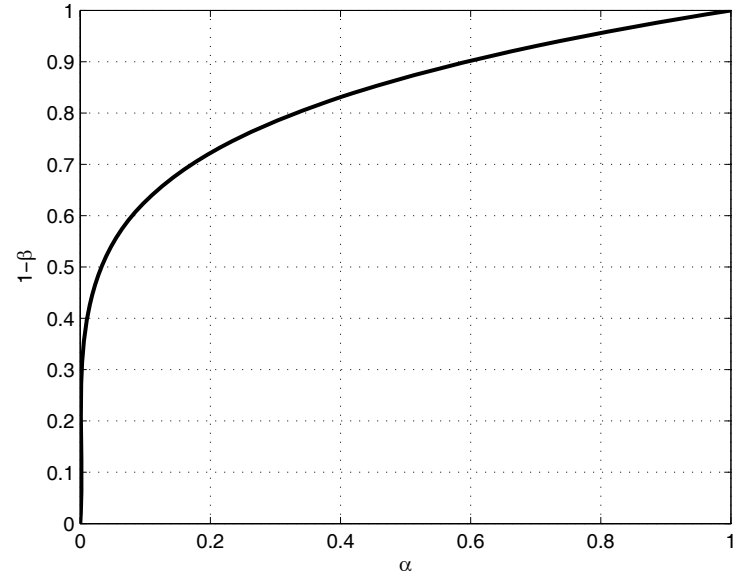

Fig. 3. ROC for the spectrum sensor in use.

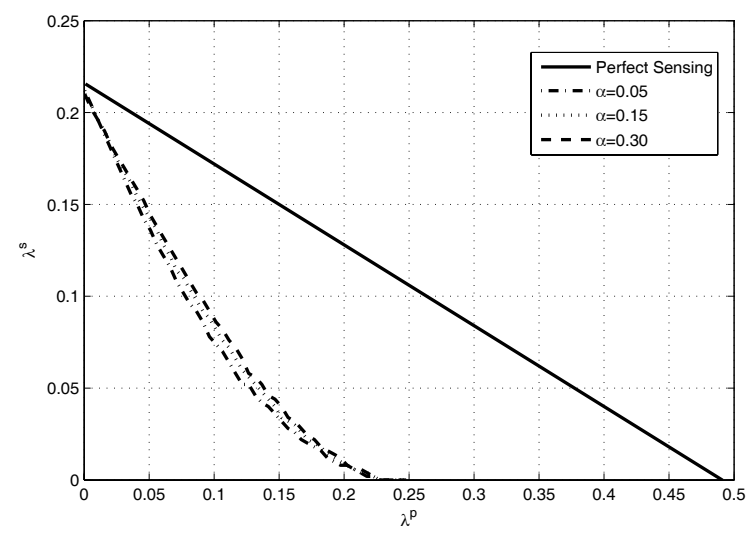

Fig. 4. Effect of sensing errors on system stability.

Fig. 3 depicts the ROC for the used spectrum sensor. It can be seen that it has moderate performance. Fig. 4 compares the stability region of the system with perfect sensing and the system with non-perfect sensing for different values of the false alarm rate $\alpha$. The negative effect of sensing errors on the stability of both primary and secondary nodes is clearly seen. For instance, for a primary arrival rate of $\lambda_{p}=0.1$, the maximum stable secondary throughput suffered a $55 \%$ reduction. Furthermore, above a primary arrival rate of $\lambda_{p}=0.23$ no secondary node can exist in the system; otherwise the whole system of queues will become unstable.

From Fig. 4 we see that by allowing the false alarm rate to increase in the detector design, a very slight improvement in secondary throughput is noticed. This is mainly because of the reduction in missed detection probability associated with the increase in the false alarm probability. By reducing the missed detection probability, primary nodes will have better service rates, hence higher probability of having empty queues and idle time slots. It is noted from (25), that the increase in false alarm rate and reduction in missed detection probability are affecting secondary throughput in opposite directions. However, the results of Fig. 4 indicates that the gains of reducing the missed detection probability outweigh the degradation due to increased false alarm rate. 


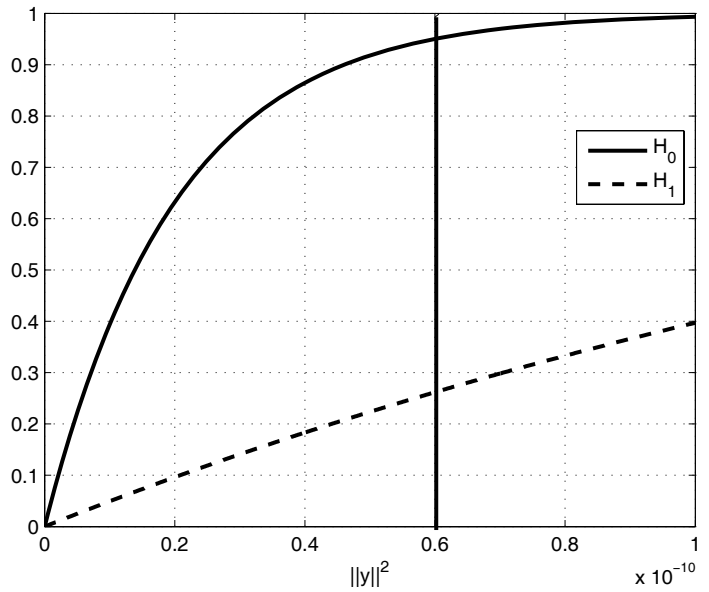

Fig. 5. CDF of the decision statistics under both hypotheses. The vertical line marks the position of the decision threshold.

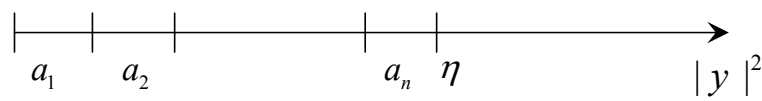

Fig. 6. Division of the interval $[0, \eta]$ into subintervals and the associated access probabilities.

\section{JOINT DESIGN OF SENSING AND ACCESS MECHANISMS}

In the previous section the detrimental effects of the errors in spectrum sensing were characterized. One of the main causes of these effects is that secondary nodes base their channel access decisions solely on the outcomes of the spectrum sensor without taking into consideration the possibility that those outcomes incorrect.

For the secondary nodes to have better channel access decisions, it is necessary to find a method with which they can assess the reliability of the spectrum sensor outcomes. Here we propose the use of the decision statistics $\left\|y_{i j}\right\|^{2}$ used by the energy detector as a measure for the reliability of the spectrum sensor decisions.

The reasoning behind the use of the value of the decision statistics is that under hypothesis $\mathcal{H}_{0}$, the value of $\left\|y_{i j}\right\|^{2}$ has a much higher probability of being closer to zero and far away from the threshold, as can be seen in Fig. 5 depicting the CDF of $\left\|y_{i j}\right\|^{2}$ under both hypotheses. Therefore, the lower the value of $\left\|y_{i j}\right\|^{2}$, the more likely hypothesis $\mathcal{H}_{0}$ is true, and the more reliable the decision is. On the other hand, as the value of the decision statistics approaches the decision threshold it is more or less equally likely that it is resulting from either one of the hypotheses. Therefore, the closer the value of $\left\|y_{i j}\right\|^{2}$ is to the decision threshold, the less reliable the outcome of the spectrum sensor is.

In order to exploit the reliability measure established above in taking channel access decisions, we propose the following scheme for channel access at the $j^{t h}$ secondary node:

- The interval $[0, \eta]$ is divided into $n$ subintervals as shown in Fig. 6.
- For each subinterval $k \in[1, n]$, assign an access probability $a_{i}^{k}$.

- Whenever the decision statistics falls in the $k^{\text {th }}$ interval, secondary node will access the channel with the associated access probability.

- In the case when $\left\|y_{i j}\right\|^{2}>\eta$, secondary node does not access the channel.

This scheme will enable us to have higher access probabilities for the subintervals closer to zero, since in these subintervals there is a very low probability of colliding with primary transmissions. Moreover, assign lower probabilities to the subintervals close to the decision threshold, where there is a higher risk of collisions.

It should be noted that under the proposed scheme, the decision threshold $\eta$ is not necessarily chosen according to the Neyman-Pearson design criterion since it is the choice of the access probabilities $a_{k}^{j}$ that will be governing the false alarm rate. However, for comparison purposes we will employ the Neyman-Pearson threshold of (14) as the threshold in our design.

\section{A. Stability Analysis}

As in the previous section, to characterize the system's stability region we need to describe the primary and secondary service processes and calculate the corresponding service rates. We start by calculating the average primary service rate $\mu_{i}^{p}$ under the proposed secondary access scheme. Since collisions between primary and secondary transmission are inevitable, the group of primary and secondary queues are interacting. Therefore, to decouple this interaction, we resort to the dominant system defined in the previous section in which secondary nodes attempt to transmit dummy packets if their queues are empty. Under this system, the service process of the $i^{\text {th }}$ primary queue will still be given by (22). The difference will be in the definition of the event that a secondary node's transmission collides with primary transmission, $\left\{\overline{\mathcal{B}_{j}^{i} \cap P_{j}^{a}}\right\}$. Here the events of missed detection and channel access are no longer independent and cannot be expressed as two separate events as above. The probability of collision is now given by $\sum_{k \in[1, n]} I_{i j}^{1 k} a_{k}^{j}$, where $a_{k}^{j}$ is user $j$ access probability associated with subinterval $k$ (see Fig. 6), and $I_{i j}^{1 k}$ is the probability that the received energy value $\left\|y_{i j}\right\|^{2}$ due to primary user $i$ transmission falls in the $k^{t h}$ subinterval when hypothesis $\mathcal{H}_{1}$ is true (primary node exists in the channel), which from the signal model of (1) is calculated as

$$
I_{i j}^{1 k}=\exp \left(\frac{(k-1) \eta}{2 n \sigma_{i j}^{2}}\right)-\exp \left(\frac{k \eta}{2 n \sigma_{i j}^{2}}\right) .
$$

Therefore, the $i^{t h}$ primary node average service rate is given by

$$
\mu_{i}^{p}=\omega_{i}^{p}\left(1-P_{i}^{o p}\right) \prod_{j \in \mathcal{M}_{s}}\left(1-\sum_{k \in[1, n]} I_{i j}^{1 k} a_{k}^{j}\right) .
$$

The $j^{t h}$ secondary node service process is still given by (24), with the exception that the probability of the event that the secondary node has access to the channel under hypothesis 
$\mathcal{H}_{0}$ is $\sum_{k \in[1, n]} I_{j}^{0 k} a_{k}^{j}$, where $I_{i j}^{1 k}$ is the probability that the received energy value $\left\|y_{i j}\right\|^{2}$ falls in the $k^{t h}$ subinterval when hypothesis $\mathcal{H}_{0}$ is true (no primary node exists in the channel), which from the signal model of (1) is calculated as

$$
I_{j}^{0 k}=\exp \left(\frac{(k-1) \eta}{2 n \sigma_{0}^{2}}\right)-\exp \left(\frac{k \eta}{2 n \sigma_{0}^{2}}\right) .
$$

Therefore, the average $j^{\text {th }}$ secondary node service rate can be written as

$$
\begin{aligned}
\mu_{j}^{s} & =\left(1-\sum_{i \in \mathcal{M}_{p}} \frac{\lambda_{i}^{p}}{\left(1-P_{i}^{o p}\right) \prod_{j \in \mathcal{M}_{s}}\left(1-\sum_{k \in[1, n]} I_{i j}^{1 k} a_{k}^{j}\right)}\right) \\
& \times\left(1-P_{j}^{o s}\right)\left(\sum_{k \in[1, n]} I_{j}^{0 k} a_{k}^{j}\right) \prod_{l \in \mathcal{M}_{s} \backslash j}\left(1-\sum_{k \in[1, n]} I_{l}^{0 k} a_{k}^{l}\right) .
\end{aligned}
$$

Define the column vector a = $\left(a_{1}^{1}, \ldots, a_{n}^{1}, a_{a}^{2}, \ldots a_{n}^{2}, \ldots, a_{1}^{M_{s}}, \ldots, a_{n}^{M_{s}}\right)^{T}$ of access probabilities for all secondary nodes, where $T$ denotes matrix transposition, the stability region of the system of primary and secondary queues can then be written as

$$
\begin{aligned}
\mathcal{R}(\mathbf{a})= & \left\{\left(\lambda_{1}^{p}, \ldots, \lambda_{M_{p}}^{p}, \lambda_{1}^{s}, \ldots, \lambda_{M_{s}}^{s}\right) \in R^{+\left(M_{p}+M_{s}\right)}:\right. \\
& \sum_{i \in \mathcal{M}_{p}} \frac{\lambda_{i}^{p}}{\left(1-P_{i}^{o p}\right) \prod_{j \in \mathcal{M}_{s}}\left(1-\sum_{k \in[1, n]} I_{i j}^{1 k} a_{k}^{j}\right)}<1, \\
& \left.\lambda_{j}^{s}<\mu_{j}^{s}, \forall j \in \mathcal{M}_{s}\right\},
\end{aligned}
$$

for $m u_{j}^{s}$ defined in (31). The maximum stability region can be determined by taking the union over all possible values of the access probability vector a as follows,

$$
\mathcal{R}=\bigcup_{\mathbf{a} \in[0,1]^{n M_{s}}}\{\mathcal{R}(\mathbf{a})\}
$$

Similar to information theoretic capacity proofs, queuing theory stability analysis is in general nonconstructive. This means that while the analysis characterizes the stability region of the system, or in other words determines the set of arrival rate vectors for which the system is stable, it does not provide any means for calculating the corresponding set of access probabilities that guarantee the system stability. In order to give insights on how our proposed access scheme can be used in the design of a cognitive radio network, we will consider the symmetric network case in the next section and provide a design method for the system in such a case.

\section{B. Symmetric Network Case}

Here we will consider the special case of a symmetric network in which it is assumed that all $M_{p}$ primary nodes have the same arrival rate $\lambda_{p}$ and all $M_{s}$ secondary nodes have the same arrival rate $\lambda_{s}$. Furthermore, it is assumed that within each network channels are also symmetric. In other words, all primary users share the same channel statistics, and all secondary users share the same channel statistics.

Under symmetry assumptions, it can be easily shown that in a TDMA network service rate is maximized when time slots are shared equally between nodes, i.e., $\omega_{i}^{p}=1 / M_{p}$. Furthermore, in an ALOHA network service rate is maximized when all nodes employ the same access probability $P_{j}^{a}=1 / M_{s}$, this property will allow us to search for a single access probability vector $\mathbf{a}=\left(a_{1}, \ldots, a_{n}\right)$ which will be shared by all secondary nodes. Equations (29) and (31) for primary and secondary service rates are now simplified to

$$
\mu_{p}=\frac{1-P^{o p}}{M_{p}}\left(1-\sum_{k \in[1, n]} I^{1 k} a_{k}\right)^{M_{s}}
$$

and

$$
\begin{aligned}
\mu_{s}= & \left(1-\frac{\lambda^{p} M_{p}}{\left(1-P^{o p}\right)\left(1-\sum_{k \in[1, n]} I^{1 k} a_{k}\right)^{M_{s}}}\right) \\
& \times\left(1-\sum_{k \in[1, n]} I^{0 k} a_{k}\right)^{M_{s}-1}\left(1-P^{o s}\right) \sum_{k \in[1, n]} I^{0 k} a_{k},
\end{aligned}
$$

where all subscripts corresponding to specific primary or secondary nodes where dropped since nodes are now indistinguishable due to symmetry.

Since the cognitive principle is based on the idea that the presence of the secondary system should be "transparent" to the primary, and since we are interested in the stable throughput of primary and secondary networks, we define the secondary system "transparency" as not affecting the primary system's stability [41]. In other words, for a given stable primary system with arrival rate $\lambda_{p}$, secondary activity will be considered transparent if the primary system maintains its stability in the presence of the secondary system. Therefore, the main design criteria for the secondary access scheme will be to maximize its own throughput under the constraint that primary stability is not affected. This design criterion can be formulated as the following constrained optimization problem

$$
\max _{\mathbf{a} \in[0,1]^{n}} \mu_{s} \text { subject to } \lambda_{p}<\mu_{p} .
$$

Fortunately, the optimization problem of (36) using (29) and (31) can be converted to a convex program. The global optimum of convex optimization problems can efficiently be obtained via standard numerical techniques [42].

To convert the optimization problem (36) given (34) and (35) into a convex program we take the logarithm, which is a monotonic function, of both the objective function and the constraint, the problem then becomes,

$$
\begin{array}{r}
\max _{\mathbf{a} \in[0,1]^{n}} \log \left(1-\frac{\lambda^{p} M_{p}}{\left(1-P^{o p}\right)\left(1-\sum_{k \in[1, n]} I^{1 k} a_{k}\right)^{M_{s}}}\right) \\
+\log \left(1-\sum_{k \in[1, n]} I^{0 k} a_{k}\right)+\log \left(\sum_{k \in[1, n]} I^{0 k} a_{k}\right) \\
+\left(M_{s}-1\right)+\left(1-P^{o s}\right)
\end{array}
$$


subject to

$$
\log \lambda_{p}<\log \left(\frac{1-P^{o p}}{M_{p}}\right)+\log \left(1-\sum_{k \in[1, n]} I^{1 k} a_{k}\right)+M_{s}
$$

except the first term in the objective all other terms are linear. To prove the convexity of that first term, let $\epsilon=\frac{\lambda^{p} M_{p}}{\left(1-P^{o p}\right)}$, where $\mathbf{I}$ is a column vector of $I^{1 k}$. The first term of the objective can then be written as

$$
1-\epsilon\left(1-\mathbf{I}^{T} \mathbf{a}\right)^{-M_{s}} .
$$

To prove convexity we will applying the rule that a function is convex if and only if it is convex when restricted to any line that intersects its domain [42]. We form the function

$$
g(t)=\log \left(1-\epsilon\left(1-\mathbf{I}^{T} \overline{\mathbf{a}}-t \mathbf{I}^{T} \mathbf{v}\right)^{-M_{s}}\right),
$$

where $t$ is a scalar parameter, $\overline{\mathbf{a}}$ belongs to the domain of the problem, and $\mathbf{v}$ is a vector such that $\overline{\mathbf{a}}+t \mathbf{v}$ also belongs to the domain of the problem. The domain is specified by the inequality constraint of the optimization problem (36) and that $\mathbf{a} \in[0,1]^{n}$.

According to the aforementioned property of convex functions, if $g(t)$ is proved to be concave with respect to $t$ (and, hence, its negative would be convex), then the function

$$
\log \left(1-\epsilon\left(1-\mathbf{I}^{T} \mathbf{a}\right)^{-M_{s}}\right)
$$

is concave with respect to a. The concavity of $g(t)$ can be easily proven via differentiating twice and examining the sign of the second derivative, which is given by

$$
\ddot{g}(t)=\frac{\epsilon M_{s}\left(\mathbf{I}^{T} \mathbf{v}\right)^{2}\left[\epsilon-\left(M_{s}+1\right)\left(1-\mathbf{I}^{T} \overline{\mathbf{a}}-t \mathbf{I}^{T} \mathbf{v}\right)^{M_{s}}\right]}{\left[\left(1-\mathbf{I}^{T} \overline{\mathbf{a}}-t \mathbf{I}^{T} \mathbf{v}\right)^{M_{s}+1}-\epsilon\left(1-\mathbf{I}^{T} \overline{\mathbf{a}}-t \mathbf{I}^{T} \mathbf{v}\right)\right]^{2}} .
$$

Since the queueing stability condition requires that $\epsilon<$ $\left(1-\mathbf{I}^{T} \overline{\mathbf{a}}-t \mathbf{I}^{T} \mathbf{v}\right)^{M_{s}}$, then

$$
\epsilon<\left(M_{s}+1\right)\left(1-\mathbf{I}^{T} \overline{\mathbf{a}}-t \mathbf{I}^{T} \mathbf{v}\right)^{M_{s}}
$$

Consequently, $\ddot{g}(t)$ is negative and $\log \left(1-\epsilon\left(1-\mathbf{I}^{T} \mathbf{a}\right)^{-M_{s}}\right)$ is concave. Concavity of the remaining terms could be proven by simply differentiating twice and examining the sign of the second derivative.

It is clear that in order to solve the optimization problem of (36) like any system that employs energy detection, knowledge of the average outage probabilities $P^{o p}$ and $P^{o s}$ and the received signal variance under the two hypotheses is required. In the assumed stationary scenario, secondary nodes will have enough time to infer these parameters during an observation cycle prior to actual system operation. Such a goal can be achieved through collaboration between secondary nodes to have good estimates of these parameters.

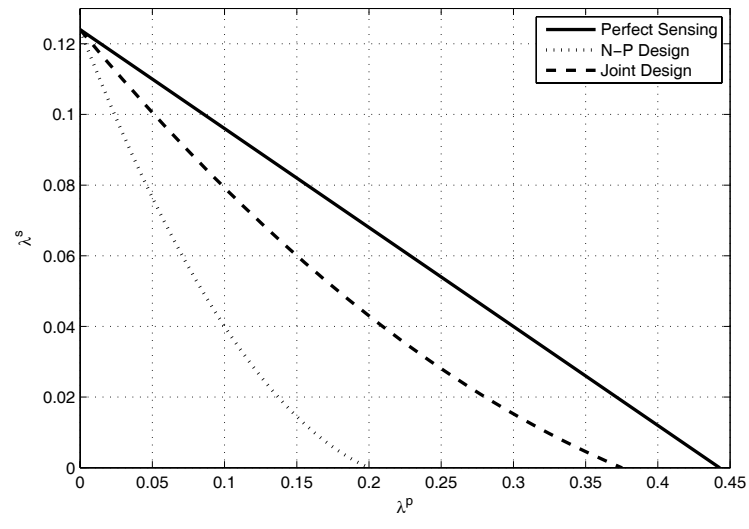

Fig. 7. Comparison between the joint design and the N-p design.

\section{Results and Discussions}

Here we compare the performance of the proposed joint design of spectrum sensing and channel access mechanisms with the conventional approach based on the Neyman-Pearson (N-P) energy detector design. First, we consider the asymmetric network case. The network will have $M_{p}=4$ primary nodes and $M_{s}=4$ secondary nodes uniformly distributed over a square region with $250 \mathrm{~m}$ edges. Primary and secondary destination are located at the center of the square region. SNR threshold is $15 \mathrm{~dB}$, transmit power is $100 \mathrm{~mW}$, path loss exponent $\gamma=3.7$, and noise power $N_{0}=10^{-11} \mathrm{~W}$. Results are obtained by averaging over 20 independent realizations. Again we plot the aggregate primary arrival rate $\lambda^{p}=\sum_{i} \lambda_{i}^{p}$ and aggregate secondary arrival rate $\lambda^{s}=\sum_{j} \lambda_{j}^{s}$.

Fig. 7 compares the stability regions for the ideal case with no sensing errors, the N-P based energy detector, and our joint design scheme with $n=4$ subintervals, using the same threshold as the one used by the N-P design given by (14). Significant improvement in the maximum stable throughput of both primary and secondary networks is observed with our proposed scheme. We see that the range of primary arrival rates for which secondary nodes can exist in the system without affecting primary stability is $85 \%$ of that of the ideal system, compared to $45 \%$ with the conventional design. Furthermore, for any given primary arrival rate the secondary system can double its arrival rate while maintaining queues' stability. This significant improvement is mainly because the joint design criteria takes the reliability of measurements into account when making access decisions rather than relying solely on the hard decision of the spectrum sensor to decide when to access the channel. This will allow the secondary node to access the channel more often when there is a high probability that no primary node is active, on the other hand, when this probability is low, secondary nodes are not allowed to access the channel, which reduces the probability of collision between primary and secondary transmissions and improving primary system's stability.

The effect of the number of subintervals $n$ used is depicted on Fig. 8. By increasing the number of subintervals, the ability to fine tune the access probability as a function of the received energy is increased. This is then translated in to an improved stability region with the increase in the number of subintervals. 


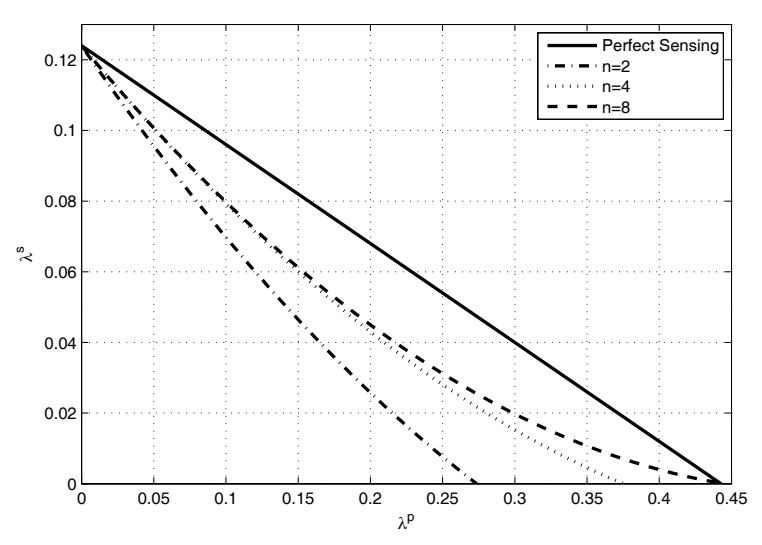

Fig. 8. Stability region for different number of subintervals $n$.

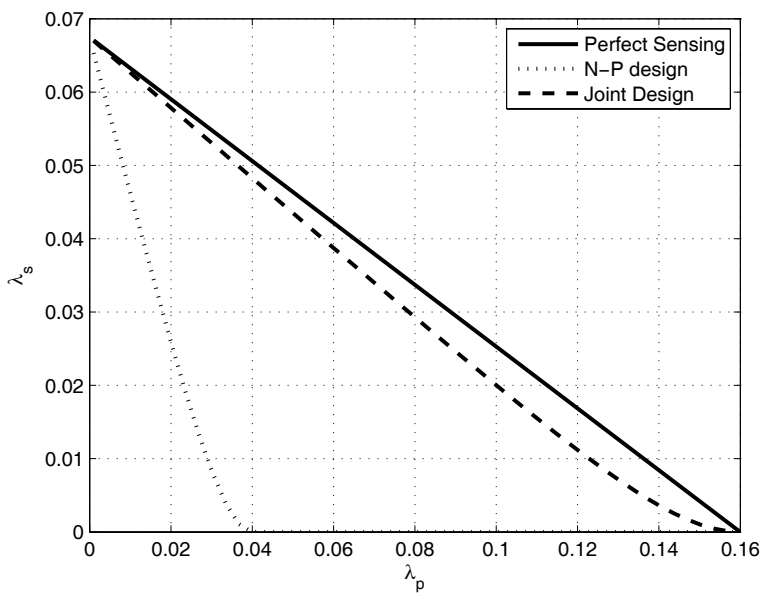

Fig. 9. Stability regions for the symmetric network case.

The results of Fig. 8 reveals that doubling $n$ from 2 to 4 has a larger effect on the performance than when doubling it from 4 to 8 . Therefore, we can conclude that a choice of $n=4$ offers a good tradeoff between the performance gain and the system complexity.

Now We consider the symmetric network case with $M_{p}=4$ primary nodes and $M_{s}=4$ secondary nodes. Distance between primary nodes and their destination is set to $100 \mathrm{~m}$, distance between secondary nodes and their destination is also $100 \mathrm{~m}$, and distance between primary and secondary nodes is $150 \mathrm{~m}$. SNR threshold is $25 \mathrm{~dB}$, transmit power is $100 \mathrm{~mW}$, path loss exponent $\gamma=3.7$, and $N_{0}=10^{-11} \mathrm{~W}$.

Fig. 9 illustrates the stability regions for the ideal case with no sensing errors, for the N-P based detector, and our joint design scheme with $n=4$ subintervals, using the same threshold as the one used by the N-P design for a false alarm rate $\alpha=0.1$. Again as in the asymmetric case, the proposed channel access scheme provides significant performance improvement in terms of stability. It is able to achieve a stability region which is at least $80 \%$ of the stability region of the perfect sensing case. This significant improvement is mainly because the proposed access scheme does not blindly rely on the outcome of the spectrum sensing operation, but also takes the reliability of the measurements into consideration.

To get more insight into how the channel access probabilities are selected, Fig. 10 depicts the channel access probabil-

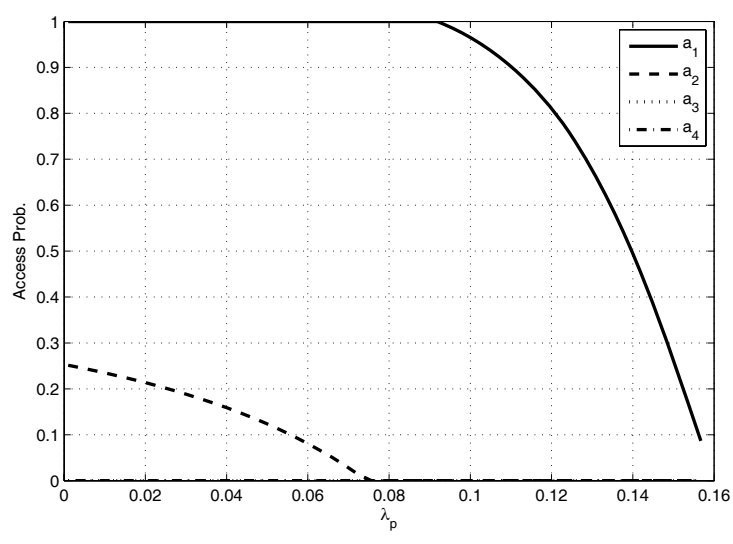

Fig. 10. Secondary access probabilities.

ities as a function of primary arrival rate. It is noted that $a_{1}$, the access probability for the interval nearest to zero, takes the highest values. This is expected since measurements that land in the corresponding interval have the highest probability of being generated when no primary users are in the channel, hence it is safe that secondary users transmit. As the primary arrival rate increases, all the access probabilities decrease to limit secondary interference to primary transmissions in order to guarantee the stability of primary queues. It is also noted that $a_{3}$ and $a_{4}$ are exactly zero for all values of $\lambda_{p}$, which means that to guarantee queues' stability transmissions in the corresponding intervals are not allowed. Furthermore, it indicates that although the choice of the threshold $\eta$ according to the N-P criterion is not optimal given our design criteria, the proposed technique was able to adapt the access probability accordingly.

\section{CONCLUSIONS}

In this paper we investigated the question of how spectrum sensing errors affect the performance of a cognitive radio networks from a MAC layer perspective. Analytical results reveal severe degradation in terms of throughput for both primary and secondary networks. The conclusion drawn is that separating the design of the spectrum sensing and the channel access mechanisms is suboptimal, and can have detrimental effects on the performance of both primary and secondary networks. Based on this observation, a joint design of spectrum sensing and channel access mechanisms was proposed and analyzed. The joint design made use of the fact that, in a binary hypothesis testing problem, the value of the test statistics could be used as a measure of how reliable the test outcome is. Analytical results of the system's performance under the proposed scheme show significant improvements in terms of the throughput of both primary and secondary networks.

\section{REFERENCES}

[1] S. Haykin, "Cognitive radio: brain-empowered wireless communications," IEEE J. Sel. Areas Commun., vol. 23, no. 2, pp. 201-220, Feb. 2005.

[2] N. Devroye, P. Mitran, and V. Tarokh, "Achievable rates in cognitive radio," IEEE Trans. Inf. Theory, vol. 52, no. 5, p. 1813-1827, May 2006.

[3] A. Jovicic and P. Viswanath, "Cognitive radio: an information-theoretic perspective," in Proc. IEEE Intl. Symp. Inf. Theory, July 2006, pp. 24132417. 
[4] Q. Zhao, L. Tong, A. Swami, and Y. Chen, "Decentralized cognitive MAC for opportunistic spectrum access in ad hoc networks: a POMDP framework," IEEE J. Sel. Areas Commun., vol. 25, p. 589-600, Apr. 2007.

[5] Y. Xing, R. Chandramouli, S. Mangold, and S. S. N., "Dynamic spectrum access in open spectrum wireless networks," IEEE J. Sel. Areas Commun., vol. 24, no. 3, p. 626-637, Mar. 2006.

[6] L. Cao and H. Zheng, "Distributed spectrum allocation via local bargaining," in Proc. IEEE Conf. Sensor Ad Hoc Commun. Netw., Sep. 2005.

[7] Z. Ji and K. J. R. Liu, "Dynamic spectrum sharing: a game theoretical overview," IEEE Commun. Mag., 2007.

[8] D. Cabric, S. M. Mishra, and R. W. Brodersen, "Implementation issues in spectrum sensing for cognitive radio," in Proc. Asilomar Conf. Signals, Syst., Comput., 2004, pp. 772-776.

[9] S. M. Kay, Fundamentals of Statistical Signal Processing: Detection Theory. Prentice Hall, 1998.

[10] H. V. Poor, An Introduction to Signal Detection and Estimation. Springer-Verlag, 1994.

[11] S. Enserink and D. Cochran, "A cyclostationary feature detector," in Proc. 28th Asilomar Conf. Signals, Syst., Comput., Oct. 1994.

[12] A. Sahai, N. Hoven, and R. Tandra, "Some fundamental limits on cognitive radio," in Proc. Allerton Conf. Commun., Control, Comput., Oct. 2004.

[13] A. Ghasemi and E. Sousa, "Collaborative spectrum sensing for opportunistic access in fading environments," in Proc. 1st IEEE Symp. New Frontiers Dynamic Spectrum Access Netw., Nov. 2005, p. 131-136.

[14] S. M. Mishra, A. Sahai, and R. W. Brodersen, "Cooperative sensing among cognitive radio," in Proc. IEEE ICC, 2006, pp. 1658-1663.

[15] E. Vistotsky, S. Kuffner, and R. Peterson, "On collaborative detection of TV transmissions in support of dynamic spectrum sharing," in Proc. IEEE Symp. New Frontiers Dynamic Spectrum Access Netw., Nov. 2005.

[16] R. S. Blum, S. A. Kassam, and H. V. Poor, "Distributed detection with multiple sensors-part II: advanced topics," Proc. IEEE, vol. 85 , no. 1 , p. 64-79, Jan. 1997.

[17] G. Ghurumuruhan and Y. Li, "Agility improvement through cooperative diversity in cognitive radio," in Proc. IEEE Global Commun. Conf., Nov. 2005.

[18] P. Papadimitratos, S. Sankaranarayanan, and A. Mishra, "A bandwidth sharing approach to improve licensed spectrum utilization," IEEE Commun. Mag., vol. 43, pp. 10-14, Dec. 2005.

[19] Y. Chen, Q. Zhao, and A. Swami, "Distributed cognitive MAC for Energy-Constrained Opportunistic Spectrum Access," in Proc. MILCOM, Oct. 2006, pp. 1-7.

[20] Q. Zhao, S. Geirhofer, L. Tong, and B. M. Sadler, "Optimal dynamic spectrum access via periodic channel sensing," in Proc. WCNC, Mar. 2007, pp. 33-37.

[21] H. Zheng and C. Peng, "Collaboration and fairness in opportunistic spectrum access," in Proc. IEEE Intl. Conf. Commun., May 2005.

[22] R. Etkin, A. Parekh, and D. Tse, "Spectrum sharing for unlicensed bands," in Proc. IEEE DySPAN 2005.

[23] Z. Ji and K. J. R. Liu, "Dynamic pricing approach for spectrum allocation in wireless networks with selfish users," in Proc. IEEE Globecom, Nov. 2006

[24] _ _ "Belief-assisted pricing for dynamic spectrum allocation in wireless networks with selfish users," in Proc. IEEE SECON 2006, Sep. 2006, pp. 119-127.

[25] K. J. R. Liu, A. K. Sadek, W. Su, and A. Kwasinski, Cooperative Communications and Networking. Cambridge University Press, 2008.

[26] Y. Xing, R. Chandramouli, S. Mangold, and S. S. N, "Dynamic spectrum access in open spectrum wireless networks," IEEE J. Sel. Areas Commun., vol. 24, no. 3, pp. 626-637, Mar. 2006.

[27] S. Keshavamurthy and K. Chandra, "Multiplexing analysis for spectrum sharing," in Proc. IEEE MILCOM'06, Oct. 2006.

[28] K. Challapali, S. Mangold, and Z. Zhong, "Spectrum agile radio: detecting spectrum opportunities," in Proc. Int. Symp. Advanced Radio Technol., Mar. 2004.

[29] B. Wild and K. Ramchandran, "Detecting primary receivers for cognitive radio applications," in Proc. 1st IEEE Symp. New Frontiers Dynamic Spectrum Access Netw., Nov. 2005, pp. 124-130.

[30] N. Benvenuto, S. Pupolin, and G. Guidotti, "Performance evaluation of multiple access spread spectrum systems in the presence of interference," IEEE Trans. Veh. Technol., vol. 37, no. 2, pp. 73-77, 1988.

[31] B. Hajek, A. Krishna, and R. O. LaMaire, "On the capture probability for a large number of stations," IEEE Trans. Commun., vol. 45, no. 2, pp. 254-260, Feb. 1997.

[32] M. Zorzi and R. Rao, "Capture and retransmission control in mobile radio," IEEE J. Sel. Areas Commun., vol. 12, pp. 1289-1298, Oct. 1994.
[33] M. Kobayashi, G. Caire, and D. Gesbert, "Impact of multiple transmit antennas in a queued SDMS/TDMA downlink," in Proc. 6th IEEE Workshop Signal Proc. Advances Wireless Commun., June 2005.

[34] W. Szpankowski, "Stability conditions for some multiqueue distributed system: buffered random access systems," Adv. Appl. Probab., vol. 26, pp. 498-515, 1994.

[35] R. W. Wolff, Stochastic Modeling and the Theory of Queues. Prentice Hall, 1989.

[36] W. Luo and A. Ephremides, "Stability of $\mathrm{N}$ interacting queues in random-access systems," IEEE Trans. Inf. Theory, vol. 45, no. 5, pp. 1579-1587, July 1999.

[37] R. M. Loynes, "The stability of a queue with non-independent interarrival and service times," Proc. Cambridge Philos. Soc., pp. 497-520, 1962.

[38] R. Rao and A. Ephremides, "On the stability of interacting queues in a multi-access system," IEEE Trans. Inf. Theory, vol. 34, pp. 918-930, Sep. 1988.

[39] V. Naware, G. Mergen, and L. Tong, "Stability and delay of finite-user slotted ALOHA with multipacket reception," IEEE Trans. Inf. Theory, vol. 51, no. 7, pp. 2636-2656, July 2005.

[40] D. Bertsekas and R. Gallager, Data Networks, 2nd edition. Prentice Hall, 1992.

[41] O. Simeone, Y. Bar-Ness, and U. Spagnolini, "Stable throughput of cognitive radios with and without relaying capability," IEEE Trans. Commun., vol. 55, Dec. 2007.

[42] S. Boyd and L. Vandenberghe, Convex Optimization. Cambridge University Press, 2004.

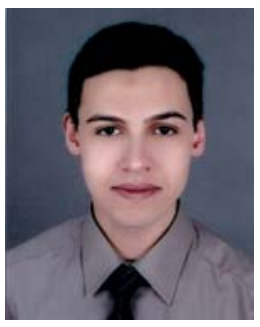

Amr El-Sherif (S' 00, M' 08) received the B.Sc. degree (with highest Honors) and the M.Sc. degree in electrical engineering form Alexandria University, Alexandria, Egypt in 2002 and 2005, respectively. $\mathrm{He}$ received the Ph.D. degree in electrical engineering from the University of Maryland, College Park, in 2009.

$\mathrm{He}$ is currently an Assistant Professor in the Electrical Engineering Department at Alexandria University, Egypt. His research interests include cooperative communications and networking, crosslayer design for wireless networks, multiple access technologies for wireless and sensor networks, and spectrum sharing and cognitive radio systems.

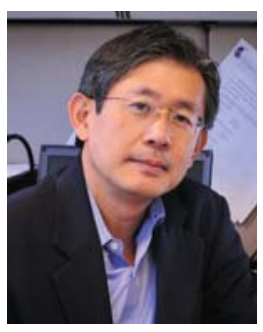

K. J. Ray Liu (F'03) is named a Distinguished Scholar-Teacher of University of Maryland, College Park, in 2007, where he is Christine Kim Eminent Professor in Information Technology. He serves as Associate Chair of Graduate Studies and Research of Electrical and Computer Engineering Department and leads the Maryland Signals and Information Group conducting research encompassing broad aspects of wireless communications and networking, information forensics and security, multimedia signal processing, and biomedical engineering.

Dr. Liu is the recipient of numerous honors and awards including IEEE Signal Processing Society Technical Achievement Award and Distinguished Lecturer. He also received various teaching and research recognitions from University of Maryland including university-level Invention of the Year Award; and Poole and Kent Senior Faculty Teaching Award and Outstanding Faculty Research Award, both from A. James Clark School of Engineering. An ISI Highly Cited Author in Computer Science, Dr. Liu is a Fellow of IEEE and AAAS.

Dr. Liu is President-Elect and was Vice President - Publications of IEEE Signal Processing Society. He was the Editor-in-Chief of IEEE Signal Processing Magazine and the founding Editor-in-Chief of EURASIP Journal on Advances in Signal Processing.

His recent books include Cognitive Radio Networking and Security: A Game Theoretical View (Cambridge University Press, 2010); Behavior Dynamics in Media-Sharing Social Networks (Cambridge University Press (to appear)); Handbook on Array Processing and Sensor Networks (IEEEWiley, 2009); Cooperative Communications and Networking (Cambridge University Press, 2008); Resource Allocation for Wireless Networks: Basics, Techniques, and Applications (Cambridge University Press, 2008); UltraWideband Communication Systems: The Multiband OFDM Approach (IEEEWiley, 2007); Network-Aware Security for Group Communications (Springer, 2007); Multimedia Fingerprinting Forensics for Traitor Tracing (Hindawi, 2005). 